\title{
Histone modification at the gene level
}

High-throughput sequencing studies are being used to characterize the genomic architectures of increasing numbers of cancer subtypes. Various cancers are known to have alterations of the chromatin modification and remodelling machineries; now, two sequencing studies have identified cancer-associated mutations in histones.

Both investigations involved the genome-scale sequencing of paediatric gliomas followed by the targeted resequencing of candidate mutant genes in additional samples. Schwartzentruber et al. sequenced the exomes of 48 paediatric glioblastomas and went on to analyse 784 samples of diverse gliomas. In a separate study, Wu et al. achieved whole-genome sequencing of seven samples of diffuse intrinsic pontine glioma (DIPG) followed by analysis of 43 DIPGs and 36 non-brainstem glioblastomas.

The two studies identified recurrent mutations in $H 3 F 3 A$, particularly in high-grade paediatric gliomas (30\% of paediatric glioblastomas and $60 \%$ of DIPGs). Consistent with this specificity, $\mathrm{Wu}$ et al. found no evidence of $H 3 F 3 A$ mutations in $>200$ paediatric tumours of other tissue types. $H 3 F 3 A$ encodes the histone variant $\mathrm{H} 3.3$, and the mutations were focused on two amino acids, K27 and G34. These sites are on the post-translationally modified histone tail region, suggesting that the mutations might alter the methylation or acetylation of $\mathrm{K} 27$ or the nearby K36. Although this hypothesis is difficult to test in clinical samples, Schwartzentruber et al. found increased levels of H3K36 trimethylation in a G34V-mutant tumour.

Do these histone mutations alter transcription? Interestingly, despite K27- and G34-mutant tumours being histologically indistinguishable, gene expression microarray analyses revealed distinct transcriptional programmes between these two mutant types. This result also indicates that these mutations have pleiotropic effects on gene expression, as might be expected from disruptions to chromatin. However, whether any tumour-promoting effects of these mutations involve the deregulated expression of a few or many cancer genes is currently unknown.

Indicating that chromatin might be disrupted at multiple levels, Schwartzentruber et al. also identified mutations in ATRX and DAXX in paediatric glioblastomas. These genes encode members of a chromatinremodelling complex that deposits H3.3 onto chromatin, and mutations in these genes have previously been identified in other cancer types. Additionally, Wu et al. found mutations in HIST1H3B - which encodes histone $\mathrm{H} 3.1$ - in $18 \%$ of DIPGs.

It remains to be deciphered why H3F3A mutations have such a propensity for high-grade paediatric gliomas, and functional studies will be required to establish whether mutant $H 3 F 3 A$ is an oncogenic driver.

\section{Darren J. Burgess}

This article is modified from the original in Nature Rev. Genet. (doi10.1038/nrg3181)

\section{ORIGINAL RESEARCH PAPERS}

Schwartzentruber, J. et al. Driver mutations in histone $\mathrm{H} 3.3$ and chromatin remodelling genes in paediatric glioblastoma. Nature 29 Jan 2012 (doi:10.1038/nature10833) |Wu, G. et al. Somatic histone $\mathrm{H} 3$ alterations in pediatric diffuse intrinsic pontine gliomas and non-brainstem glioblastomas. Nature Genet. 29 Jan 2012

(doi:10.1038/ng.1102)

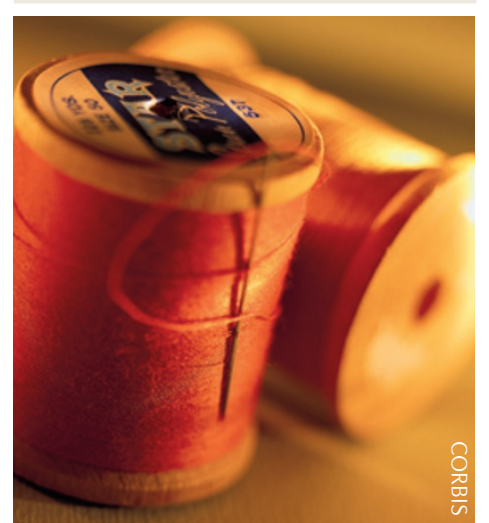

